\title{
Giant Lamb shift of atoms near lossy multimode optical micro-waveguides
}

\author{
Peter HoraK ${ }^{1,2}$, Peter Domokos ${ }^{1}\left(^{*}\right)$ and Helmut Ritsch ${ }^{1}$ \\ 1 Institut für Theoretische Physik, Universität Innsbruck, Technikerstraße 25, A-6020 \\ Innsbruck, Austria \\ 2 Optoelectronics Research Centre, University of Southampton, Southampton SO17 1BJ, \\ United Kingdom
}

\begin{abstract}
Atoms coupled to optical fields strongly confined in two spatial dimensions, as in solid state microstructures, can experience very large Lamb shifts due to a spectrally strongly asymmetric mode density. We use the generic example of a quasi one-dimensional waveguide structure driven close to cutoff frequency of a new transverse branch of propagating modes. We analytically find strong shifts of the atomic resonance frequency due to the modified vacuum, which can be an order of magnitude larger than the atomic linewidth. At the same time one gets significantly enhanced scattering of the guided light by the atom, which could be used as a tool to investigate these effects or to build non-destructive single atom detectors.
\end{abstract}

In the past years we have seen spectacular advances in our ability to cool atoms to nanoKelvin temperatures and control their motional degrees of freedom down to the quantum level [1]. In parallel, the miniaturization of optical microstructures has reached the level, where fabrication almost at the atomic scale is feasible [2]. One of the great goals in the near future is to bring together these technologies in a generation of integrated optical quantum devices [3]. As a central point to utilize such devices, we must understand the behaviour and transport of the atomic matter subject to subwavelength-structured electromagnetic radiation fields. Evanescent fields created by tailored dielectric microstructures are of primary interest in this research. A series of experiments have demonstrated the usefulness of evanescent optical fields to realize atom mirrors [4] or quasi 2D surface traps [5]. However, these surface setups have all been based on macroscopic fields regarding the transverse spatial dimension and photon numbers involved. Hence a single particle has almost no effect on the field and a single photon field would give only a negligible force on an atom.

In this Letter we show that the effective atom-photon interaction can be strongly enhanced for atoms coupled to fields of certain dielectric microstructures. It is well known that for 3D confined fields as in cavity QED setups, strong atom field coupling can be achieved [6]. Here

(*) On lcave from: Rescarch Institutc for Solid Statc Physics and Optics, Hungarian Acadcmy of Scicnces, Hungary 
we reveal new surprising phenomena beyond the single mode strong coupling limit, considering atoms interacting with a whole continuum of travelling wave modes, where the field is confined in only one or two transverse dimensions [7]. If the atomic resonance is close to the cutoff frequency of a new transverse branch of propagation modes, the modified vacuum can induce a huge atomic frequency shift exceeding the natural linewidth by orders of magnitude. In conjunction with this large Lamb shift, a strongly increased photon scattering rate by a single atom takes place, which could be used for position and state selective single atom detection and manipulation schemes [8] as well as for very low intensity photonic devices.

Atomic Lamb shift in $1 D$ continua of modes. - Let us consider a two-level atom with resonance frequency $\omega_{a}$ in (or close to) a dielectric medium with refractive index $n_{0}$ [9], which is assumed to be infinitely extended into the $z$ direction but with a transverse dimension of the order of an optical wavelength. This microstructure supports a continuum of optical modes described by annihilation operators $a_{n}(k)$, where $n$ labels the transverse mode index and $k$ the longitudinal wave number. The annihilation and creation operators fulfill the standard commutation relation

$$
\left[a_{n}(k), a_{n^{\prime}}^{\dagger}\left(k^{\prime}\right)\right]=\delta_{n n^{\prime}} \delta\left(k-k^{\prime}\right) .
$$

The corresponding frequencies are denoted by $\omega_{n}(k)$ and the mode functions read $f_{n}(k, \mathbf{x})=$ $\exp (i k z) f_{n}^{(T)}(k, x, y)$. The mode functions are normalized such that

$$
A=n_{0}^{2} \int_{A_{1}} d x d y\left|f_{n}^{(T)}(k, x, y)\right|^{2}+\int_{A_{2}} d x d y\left|f_{n}^{(T)}(k, x, y)\right|^{2},
$$

where the first integral goes over the part of the mode function inside and the second integral over the part outside the dielectric medium. $A$ is the cross section of the microstructure. The positive frequency part of the electric field is then given by

$$
E^{(+)}(\mathbf{x})=\sum_{n} \int d k E_{0}\left(\omega_{n}(k)\right) f_{n}(k, \mathbf{x}) a_{n}(k)
$$

with $E_{0}(\omega)=\sqrt{\hbar \omega /\left(2 \epsilon_{0} A\right)}$ the electric field of a single photon. (For simplicity we neglect polarization issues here, as polarization should at least qualitatively not change our results.)

In the following we will assume that only modes with frequencies close to the atomic resonance frequency $\omega_{a}$ contribute to the dynamics. We will thus replace $E_{0}\left(\omega_{n}(k)\right)$ by the corresponding value at the atomic frequency $E_{0}=E_{0}\left(\omega_{a}\right)$ and take it out of the integral in Eq. (3). In dipole and rotating wave approximation the system dynamics is then governed by the Hamiltonian

$$
\begin{aligned}
H & =\omega_{a} \sigma^{\dagger} \sigma+\sum_{n} \int d k \omega_{n}(k) a_{n}^{\dagger}(k) a_{n}(k) \\
& +i g \sum_{n} \int d k\left[f_{n}^{*}\left(k, \mathbf{x}_{a}\right) a_{n}^{\dagger}(k) \sigma-\sigma^{\dagger} f_{n}\left(k, \mathbf{x}_{a}\right) a_{n}(k)\right] .
\end{aligned}
$$

Here $\sigma$ is the atomic lowering operator, $\mathbf{x}_{a}$ is the position of the atom, and $g=\mu E_{0}$ where $\mu$ is the atomic dipole moment. The first line of Eq. (4) describes the free field evolution and the second line the atom-light coupling.

From this Hamiltonian we obtain Heisenberg equations of motion for the operators $\sigma$ and $a_{n}(k)$, where we include photon losses from the dielectric medium at a rate $2 \kappa$ and spontaneous decay of the atomic excited state into the free space vacuum modes or by non-radiative channels, e.g., due to atom-phonon coupling, at a rate $2 \Gamma$. Next, we formally integrate the 


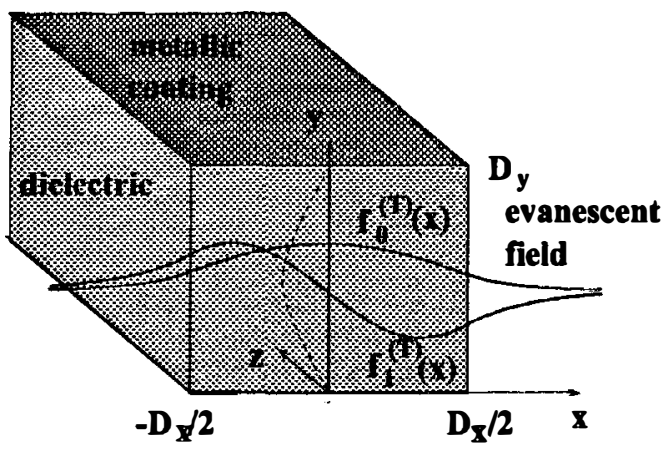

Fig. 1 - Schematic presentation of the sample microstructure.

equation for $a_{n}(k)$ and insert the solution into the equation for $\sigma$. Up to first order of the perturbation series in the small parameter $g^{2} /\left(\omega_{a} \kappa\right)$, the resulting Heisenberg equation for the atomic polarization $\sigma$ is equivalent to

$$
\frac{d}{d \dot{t}} \sigma=\left(-i \omega_{a}-\Gamma+\sum_{n} L_{n}\right) \sigma
$$

where

$$
L_{n}=-\gamma_{n}+i \delta_{n}=g^{2} \int d k \frac{\left|f_{n}\left(k, \mathbf{x}_{a}\right)\right|^{2}}{i \omega_{a}-i \omega_{n}(k)-\kappa} .
$$

Note that we assumed the limit of small atomic saturation and neglected quantum noise terms (related to spontaneous decay [10]) here. Moreover, we will in the following use the vacuum value for the decay rate $\Gamma$, which is tantamount to neglecting non-radiative decays as well as the change of the mode density of the free space (unbound) light modes due to the presence of the microstructure.

Equations (5), (6) show that the coupling of the atom to the various branches of transverse modes gives rise to atomic Lamb shifts $\delta_{n}$ and line broadenings $\gamma_{n}$. Depending on the shape of the dispersion relation $\omega_{n}(k)$, the Lamb shift and the line broadening obtained from the coupling of the atom to continuous one-dimensional sets of electric modes can significantly alter the atomic dynamics, as we will demonstrate in the remaining part of this Letter with a specific example. Since dielectric microstructures can be fabricated with high accuracy, this offers the possibility to tailor the atom-light interaction to a large extent.

Specific example of a microstructure.- In order to discuss these effects quantitatively in more detail, we will in the following concentrate on a specific example of such a microstructure.

Let us consider a dielectric medium with a rectangular cross section of height $D_{x}$ and width $D_{y}$ infinitely extended in the $z$ direction as sketched in Fig. 1 . The light is confined within this structure in the two transverse directions by two different mechanisms. In $x$ direction total internal reflection at the dielectric-vacuum interface is responsible for confinement, while in the $y$ direction confinement is provided by reflecting metallic coatings. Note, however, that this choice only serves for simplification of the calculation and is of no essential importance for the effect discussed here. $D_{x}$ and $D_{y}$ are chosen such that the structure supports only one wave number $k_{y}=\pi / D_{y}$ in the $y$ direction for the interesting range of frequencies, but is close to threshold for the existence of a first excited branch of modes in the $x$ direction.

In Fig. 2 we show the numerically calculated frequencies and longitudinal wave numbers of the modes supported by such a structure. The width $D_{y}$ is chosen such that the threshold 




Fig. 2 - Mode frequency $\omega_{n}(k)$ versus longitudinal wave number $k$. The parameters are $n_{0}=1.5$, $D_{x}=\lambda_{p} / \sqrt{n_{0}^{2}-1}$, and $D_{y}$ is chosen such that the threshold of the first excited branch in $x$ direction is $\omega_{a}$.

of the first excited branch coincides with the atomic resonance frequency, $\omega_{1}(0)=\omega_{a}$. Hence, for all modes in this branch $\omega_{1}(k) \geq \omega_{a}$. Therefore, all modes contribute with the same sign to the imaginary part of the integral in Eq. (6) and a large Lamb shift $\delta_{1}$ can be expected. Simultaneously, at threshold we have $\frac{\partial \omega_{1}(k)}{\partial k}=0$ and therefore a large number of modes contributes resonantly to the real part of the integral, that is, to the line broadening $\gamma_{1}$. Similar threshold effects are present in photonic bandgap materials, where the three dimensional vacuum is restructured such that no radiation mode exists in a certain frequency range [11]. However, here the multi-branch structure of the dispersion relation of Fig. 2 becomes essential, as the fundamental branch of propagating modes serves for resonantly driving the atom and for observing a stationary scattering scenario in an input-output system.

An analytic approximation of Eq. (6) can be obtained. The transverse part of the modes is approximated by a unique function $f_{n}^{(T)}(x, y)$ for all the relevant frequencies within each mode branch, i.e. we assume constant values of $k_{x, n}$ and a constant transverse wave number

$$
q_{n}=\frac{1}{n_{0}} \sqrt{k_{x, n}^{2}+k_{y}^{2}}
$$

Introducing an exponential convergence factor to cut off high frequencies which in the dipole approximation leads to an unphysical logarithmic divergence (missing in the minimal coupling scheme), we find by complex contour integration

$$
L_{n}=-2 \pi \frac{g^{2} n_{0}}{c}\left|f_{n}^{(T)}\left(x_{a}, y_{a}\right)\right|^{2} \sqrt{1+\left(\frac{q_{n}}{r_{n}+i s_{n}}\right)^{2}} .
$$

Here

$$
\begin{aligned}
& r_{n}=\frac{1}{c}\left[-u_{n}+\sqrt{u_{n}^{2}+\kappa^{2} \omega_{a}^{2}}\right]^{1 / 2} \\
& s_{n}=\frac{\kappa \omega_{a}}{c^{2}} \frac{1}{r_{n}} \\
& u_{n}=\frac{1}{2}\left(c^{2} q_{n}^{2}+\kappa^{2}-\omega_{a}^{2}\right) .
\end{aligned}
$$






Fig. 3 - Lamb shift $\delta_{1}$ (solid line) and level broadening $\gamma_{1}$ (dashed) due to the interaction of the atom with the first branch of excited modes versus threshold frequency $\omega_{t h}$. The atomic parameters correspond to the $D_{2}$ line of $\mathrm{Rb}, \kappa=0.001 \omega_{a}, n_{0}=1.5, D_{x}=\lambda_{p} / \sqrt{n_{0}^{2}-1}$. The atomic position is $\left(x_{a}, y_{a}, z_{a}\right)=\left(D_{x} / 2, D_{y} / 2,0\right)$, i.e., the point of maximum coupling at the surface of the dielectric medium. The plotted range of $\omega_{t h}$ corresponds to a variation of about $10 \%$ of the spatial width $D_{y}$.

For the parameters used in this Letter we compared these results with numerical integrations of Eq. (6) and found excellent agreement.

As a central result of our calculations we show the Lamb shift and photon scattering rate in Fig. 3. These can exceed the natural atomic linewidth by far. The origin of this dramatic effect on $\delta_{1}$ and $\gamma_{1}$ lies in the appearance of the first (transversally) excited mode branch and varies strongly as a function of its threshold frequency $\omega_{t h}$. For the given parameters the frequencies are well above the threshold of the lowest branch and its contribution to the Lamb shift $\delta_{0}$ and level broadening $\gamma_{0}$ are below one atomic linewidth and thus much smaller.

For $\omega_{a}>\omega_{t h}$, travelling wave solutions exist in the excited branch of modes at the atomic frequency, and the dominant effect is enhanced spontaneous atomic decay by emission of photons into the microstructure modes. For $\omega_{a}<\omega_{t h}$, no travelling solutions exist and the main effect is a shift of the atomic frequency by $\delta_{1}$. At threshold and assuming $\kappa \ll \omega_{a}$, Eq. (8) can be approximated by

$$
L_{1}=2 \pi \frac{g^{2} n_{0}}{c}\left|f_{1}^{(T)}\left(x_{a}, y_{a}\right)\right|^{2} \frac{i-1}{2} \sqrt{\omega_{a} / \kappa} .
$$

Hence, the maximum possible Lamb shift is determined by the ratio of the optical frequency to the photon loss rate from the microstructure. Thus, for our specific example the limiting factor is the reflectivity of the metallic coatings on the dielectric surfaces. For example, a reflectivity of $99 \%$ yields a decay rate $\kappa \approx \omega_{a} / 1000$ as used in Fig. 3 . Note that the magnitude as well as the scaling of the calculated Lamb shift are very different from the ones found for atoms between metallic plates [12] or in photonic band gap materials [11].

As a complementary effect of the large Lamb shift, the strong coupling leads to unusually enhanced scattering of photons by the atom. Let us calculate the scattered radiation field in the following. To this end, we introduce an additional term in the Hamiltonian (4) which describes the coupling of a single travelling wave with wave number $k_{0}$ and frequency $\omega_{p}$ of the lower branch of modes to an external driving field. With the same simplifications as used to obtain Eq. (8) and assuming $\kappa \gg\left|\Delta_{a}\right|$ (where $\Delta_{a}=\omega_{p}-\omega_{a}$ is the atom-pump detuning) 
we find the following stationary electric field (3):

$$
\begin{aligned}
E(\mathbf{x}) & \propto e^{i k_{0} z} f_{0}^{(T)}(x, y) \\
& -L_{0} \frac{e^{n_{0}\left(i r_{0}-s_{0}\right)|z|}}{i \Delta_{a}-\Gamma+\sum_{n} L_{n}} f_{0}^{(T)}(x, y) \\
& -L_{1} \frac{e^{n_{0}\left(i r_{1}-s_{1}\right)|z|} f_{0}^{(T)}\left(x_{a}, y_{a}\right)}{i \Delta_{a}-\Gamma+\sum_{n} L_{n} f_{1}^{(T)}\left(x_{a}, y_{a}\right)} f_{1}^{(T)}(x, y),
\end{aligned}
$$

where the first line gives the field of the single pumped mode, the second line is the field of the light scattered into the lower branch of modes, and the third line is the field scattered into the upper branch. As an example we plot the field intensity along the $z$ direction at the center of the medium, Fig. 4(a), and on the surface, Fig. 4(b), for an atom sitting at the surface position $\left(x_{a}, y_{a}, z_{a}\right)=\left(D_{x} / 2, D_{y} / 2,0\right)$

At the center of the structure all excited branch modes have zero amplitude and the electric field is formed by the lower branch only. Since the corresponding Lamb shift $\delta_{0}$ is small, the change of the electric field is small too. However, we see that the atom (at position $z=0$ ) scatters some light from the pumped mode into its degenerate counter-propagating mode. Hence, on top of the constant intensity of the pumped mode, there appears a standing wave with about $3 \%$ modulation on one side of the atom. Due to the damping of the light modes, this standing wave has an exponentially decaying envelope with a decay distance of $d_{0}=c /\left(n_{0} \kappa\right)$. The electric field at the surface, on the other hand, is dominated by the large Lamb shift $\delta_{1}$ due to the excited branch of modes and therefore has a much larger change of amplitude, see Fig. 4(b). Similar enhancement of light scattering has been predicted for a dielectric wire in a metallic wave guide [13]. According to Eq. (13), we find again an exponential decay with an approximate decay length of $d_{1}=c /\left(n_{0} \sqrt{\kappa \omega_{a}}\right)$, which is much shorter than that of Fig. 4(a). A spatially resolved detection of the photons lost through the coatings of the dielectric structure would thus reveal the change of field intensity and could serve as an implicit measurement of the large atomic Lamb shift. An estimate for the parameters of Fig. 4 and assuming an atomic saturation parameter of 0.1 yields a loss of the order of $10^{3}$ photons per $\mu \mathrm{m}$ length and per $\mu$ s time through the metallic coatings, which should easily be detectable.

An interesting option to detect the frequency shift can come from an atom-optical experiment where a cloud of cold atoms is dropped onto the microstructure and reflected by the evanescent light field. If the atomic cloud is dilute enough such that the mean distance between the atoms is larger than $d_{1}$, each atom will be scattered individually and the reflection of the cloud will essentially be specular. On the other hand if atoms are closer than $d_{1}$, they will interact with a distorted light field as shown in Fig. 4(b). Since the modulation of the light intensity along $x$ is roughly of the order of the total intensity and since the periodicity is of the order of an optical wavelength, the forces along $x$ will be comparable with the force in $z$ direction. Hence the average reflection of a cloud of atoms will be highly diffusive in this regime.

Conclusions.- We discussed the drastic changes of the interaction of an atom with a quasi1D optical waveguide, if one operates the system close to a branch cutoff. Although developed on a specific example, our theory is easily applicable to atom-photon interactions for a broad variety of optical microstructures. The results should hold as long as the dispersion relation for the modes has the very general shape depicted in Fig. 2. Since the mode density can be tailored much better by such structures than by Fabry-Perot cavities, the strong enhancement of the atom-photon interaction could lead to a completely new generation of cavity QED 

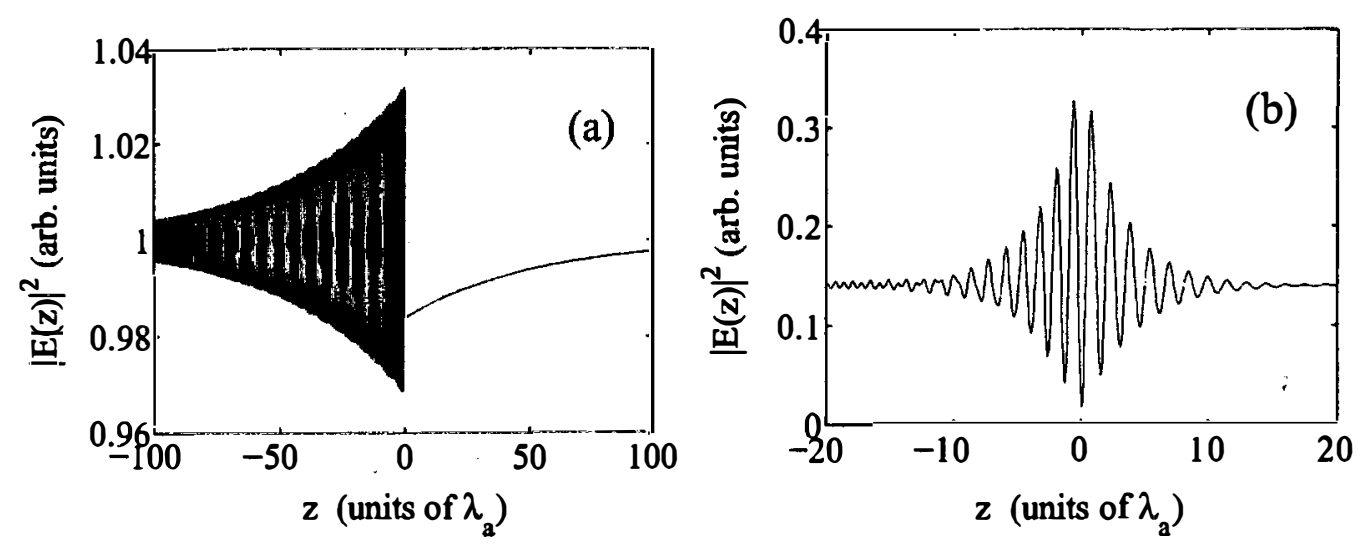

Fig. 4 - Stationary light field intensity $|E(z)|^{2}$ along $z$ for $y=D_{y} / 2$ and (a) $x=0$, (b) $x=D_{x} / 2$ in the case of a single driven mode. Parameters as in Fig. 3 with $\omega_{t h}=\omega_{p}, \Delta_{a}=10 \Gamma$.

experiments [14] with possible applications such as micro-optical devices involving only very few atoms and photons. This could have a similar impact on the field as the advent of quasi 2D or 1D electron systems with tailorable mode densities in semiconductor devices.

This work was supported by the Austrian Science Foundation FWF (project P13435-TPH) and the British Engineering and Physical Sciences Research Council EPSRC. P. D. acknowledges the financial support by the NSF of Hungary (OTKA F032341).

\section{REFERENCES}

[1] Proceedings of the International School of Physics Enrico Fermi, Vol. 140, edited by M. Inguscio, S. Stringari, and C. Wieman (IOS Press, Amsterdam, 1999).

[2] H. P. Herzig (Ed.), Micro-Optics (Taylor \& Francis, London, 1997).

[3] R. Folman et al., Phys. Rev. Lett. 84 (2000) 4749; W. Hänsel et al., Phys. Rev. Lett. 86 (2001) 608; G. Birkl et al., Opt. Commun. 191 (2001) 67.

[4] A. Landragin et al., Phys. Rev. Lett. 77 (1996) 1464; M. Gorlicki et al., Phys. Rev. A 61 (2000) 013603; F. Shimizu, Phys. Rev. Lett. 86 (2001) 987.

[5] Yu. B. Ovchinnikov, I. Manek, and R. Grimm, Phys. Rev. Lett. 79 (1997) 2225; H. Gauck et al., Phys. Rev. Lett. 81 (1998) 5298.

[6] M. Brune et al., Phys. Rev. Lett. 72 (1994) 3339.

[7] P. Domokos, P. Horak, and H. Ritsch, Phys. Rev. A 65 (2002) 033832.

[8] P. Domokos, M. Gangl, and H. Ritsch, Opt. Commun. 185, 115 (2000); P. Domokos and H. Ritsch, Europhys. Lett. 54 (2001) 306.

[9] As in most theoretical work, we are using the simplest case of a two-level atom here. Note, however, that in certain situations this is known to give incorrect Lamb shifts, see e.g. G. Barton, J. Phys. B 7 (1974) 2134.

[10] C. W. Gardiner and P. Zoller, Quantum Noise, 2nd edition (Springer-Verlag, Heidelberg, 2000).

[11] P. Lambropoulos et al., Rep. Prog. Phys. 63 (2000) 455.

[12] M. Marrocco, M. Weidinger, R. T. Sang, and H. Walther, Phys. Rev. Lett. 81 (1998) 5784; G. Barton, Proc. R. Soc. Lond. A 410 (1987) 141.

[13] R. Gómez-Medina et al., Phys. Rev. Lett. 86 (2001) 4275.

[14] E. A. Hinds, K. S. Lai, and M. Schnell, Phil. Trans. Royal Soc. London A 355 (1997) 2353. 